\section{The First Isolation of Photobacterium damselae subsp. damselae from Asian Seabass Lates calcarifer}

\author{
Praparsiri Kanchanopas-Barnette ${ }^{1}$, Alejandro \\ Labella $^{2}$, Carmen M. Alonso ${ }^{2}$, Manuel \\ Manchado $^{3}$, Dolores Castro ${ }^{2}$ and \\ Juan J. Borrego ${ }^{2 *}$ \\ ${ }^{1}$ Department of Aquatic Sciences, Faculty of Sciences, \\ Burapha University, Bangsaen, \\ Chon Buri 20131, Thailand \\ ${ }^{2}$ Department of Microbiology, Faculty of Sciences, \\ University of Malaga, Campus Teatinos, \\ 29071-Malaga, Spain \\ ${ }^{3}$ Centro El Toruño, IFAPA, Junta de Andalucía, \\ 11500-El Puerto de Santa María, \\ Cádiz, Spain
}

(Received April 4, 2008)

ABSTRACT-After transporting Asian seabass Lates calcarifer from a fish farm to the Burapha University facilities, they began to die. The affected or moribund fish exhibited abdominal swelling, yellowish decoloration around the anus, darkening of the gills and exophthalmic eyes. The internal signs of the disease included abdominal cavities filled with a cloudy yellow and gelatinous fluid together with liver hemorrhage. Isolated bacteria from several organs were biochemically characterized as Photobacterium damselae subsp. damselae. It was further confirmed by PCR. An infectivity experiment by intraperitoneal injection with an isolate LCA 24907 showed its virulence to Asian seabass with $\mathrm{LD}_{50}$ of $8.1 \times 10^{5} \mathrm{CFU} / \mathrm{g}$ fish body weight. This is the first report of isolating $P$. damselae subsp. damselae from Asian seabass.

Key words: Photobacterium damselae subsp. damselae, Lates calcarifer, Asian seabass

Photobacterium damselae subsp. damselae has been recognized as a bacterial pathogen in a wide variety of aquatic animals including both shrimps and fish ${ }^{1-6)}$. This microorganism is an autochthonous inhabitant of aquatic ecosystems, which may survive in seawater and sediment for a long time, maintaining its infectivity and pathogenic properties ${ }^{7-9}$. In addition, $P$. damselae

\footnotetext{
* Corresponding author

E-mail: jjborrego@uma.es
}

subsp. damselae is also associated with wound infections in humans ${ }^{10-12)}$

Asian seabass Lates calcarifer is the most important fish species in the marine aquaculture system in Thailand. Microbial outbreaks in juvenile Asian seabass have been reported to be caused by members of the genus Vibrio, particularly Vibrio anguillarum-like (VAL) bacteria $^{13)}$. In Thailand, shrimp and seabass are cultured alternately in the marine farms, as an attempt to prevent the spread of potential microbial pathogens. However, this strategy could be not effective for those bacterial pathogens that possess the ability to infect both shrimp and fish, like $P$. damselae subsp. damselae.

In the present study, the causative agent of the abdominal swelling affecting juvenile farmed Asian seabass was characterized by biochemical and molecular techniques. In addition, the virulence of an isolate was also established.

\section{Materials and Methods}

Five hundred Asian seabass fingerlings were obtained from a commercial fish farm in Chon Buri province (Thailand). Fish weight ranged between 30-50 g. Animals were transported to the Burapha University facilities, where they were stocked and reared in a $10 \mathrm{~m}^{3}$ tank containing brackish water at the optimal conditions of culture.

Asian seabass were reared for 20 days and observed daily. Any individuals that were dead or had abdominal swelling were removed of the tank, and external lesions were scored. Dissections were performed in aseptic conditions on all dead or affected fish. The later were previously anaesthezid with MS-222 in seawater as a final concentration of $65 \mathrm{mg} / \mathrm{mL}$, prior to sampling for microbial isolation.

The surviving affected fish were treated 5 consecutive days with chloramphenicol (100 mg/kg body weight), from the fourth day after the onset of dropsy symptoms. Seabass specimens were monitored daily during antimicrobial drug administration.

Bacterial cultures were carried out from kidney, liver and spleen tissues of each dead or moribund fish, using Tryptic Soy agar and broth (Difco, USA) supplemented with $2 \% \mathrm{NaCl}$ (TSAS and TSBS) and Marine agar (Difco). Inoculated agar plates were incubated at $25^{\circ} \mathrm{C}$ for $48 \mathrm{~h}$. Bacterial isolates were later purified on TSAS plates and TCBS (Oxoid, UK) agar supplemented with $1.5 \% \mathrm{NaCl}$ (TCBS-1), and the pure cultures were grown into TSBS. All the bacterial isolates were subjected to taxonomical analysis using the biochemical tests described in Bergey's Manual of Determinative Bacteriology ${ }^{14)}$, and the API 20E system kit (Biomerieux, France). Bacterial isolates were stored in $15 \%$ glycerol and kept at $-80^{\circ} \mathrm{C}$ for further molecular identification. For comparative purposes, $P$. damselae subsp. 
damselae ATCC 33539, CECT 5064 and P. damselae subsp. piscicida ATCC 17911 were used.

The hemolytic activity of the bacterial isolates was determine on blood agar containing 5\% human blood and incubated at $25^{\circ} \mathrm{C}$ for $24-48 \mathrm{~h}$. Hemolysis was observed by the presence of clearing in the blood around the colony.

A PCR technique, using two primer pairs directed to internal regions of the $16 \mathrm{~S}$ rRNA and ureC genes ${ }^{15}$, was employed to confirm $P$. damselae subsp. damselae.

The $50 \%$ lethal dose $\left(\mathrm{LD}_{50}\right)$ of isolated $P$. damselae subsp. damselae strain LCA 24907 was determined using healthy seabass specimens with average weights $3.7 \pm 0.6 \mathrm{~g}$, collected from a different fish farm without symptomatology evidence. Prior to the trials, $10 \%$ of the fish population were euthanized and confirmed for the absence of bacterial growth, using the primary isolation method described above. The remaining fish were divided into groups of five fish and injected intraperitoneally (IP) with bacterial dose ranging from $10^{2}$ to $10^{8}$ colony forming units $(\mathrm{CFU}) / \mathrm{mL}$ and kept at $28^{\circ} \mathrm{C}$ for 14 days. Bacteriological analyses were carried out from all dead specimens to confirm the cause of death.

\section{Results and Discussion}

Signs of illness on fish appeared 5 days after transferring the seabass from the hatchery company to the Burapha University facilities. Mortalities were observed during 10 days reaching a cumulative mortality of $49.2 \%$. The mortality rate decayed after chloramphenicol treatment, although it did not ceased until 8 days later.

Gross external characteristics of the moribund fish were swollen abdomens (Fig. 1), yellow color around the anus, darkening of the gills and exophthalmic eyes. Internally, the abdominal cavities were filled with cloudy yellow gelatinous fluid, and most fish showed liver hem-

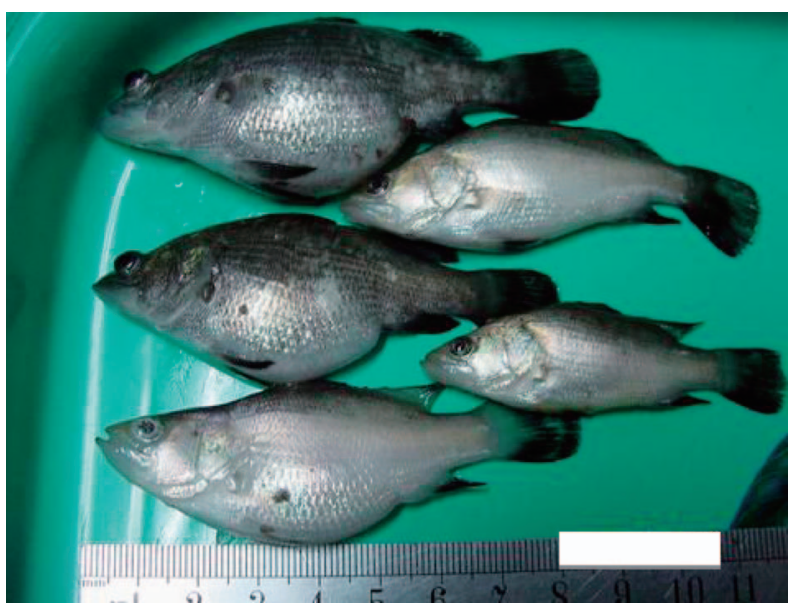

Fig. 1. Moribund Asian seabass showing dropsy abdomen and exophthalmic eyes. orrhage. These gross disease signs were similar to those previously described for vibriosis and pasteurellosis in several fish species ${ }^{2,16-18)}$, except the typical white tubercles in the spleen provoked by $P$. damselae subsp. piscicida.

Bacterial growth in pure culture on TSAS and Marine agar plates were obtained from the kidneys of all dead or moribund fish. The bacterial isolates showed a typical green-pigmented colonies on TCBS-1, which indicates inability to sucrose fermentation. Further bio-

Table 1. Comparison of biochemical and phenotypic characteristics of Asian seabass isolate LCA 24907, $P$. damselae subsp. damselae reference strain ATCC 33539 and $P$. damselae subsp. piscicida reference strain ATCC 17911

\begin{tabular}{cccc}
\hline & Asian seabass & P. damselae & P. damselae \\
Test & isolate & damsp. & subsp. \\
& LCA 24907 & ATCC 33539 & ATCC 17911 \\
& & & ATCida \\
\hline
\end{tabular}

\begin{tabular}{l} 
Motility \\
Cytochrome-oxidase \\
Nitrate reduction \\
Gas from glucose \\
Acetoine production \\
Indole production \\
O/F test \\
Urease \\
$\beta$-galactosidase \\
Lysine decarboxylase \\
Arginine dihydrolase \\
Ornithine decarboxylase \\
Gelatin liquefaction \\
Esculine hydrolysis \\
Amylase production \\
Hemolysin production \\
Citrate \\
Acid/from: \\
D-glucose \\
Lactose \\
Mannitol \\
Sorbitol \\
Inositol \\
Arabinose \\
Rhamnose \\
Sucrose \\
Melibiose \\
Growth at: \\
$4{ }^{\circ} \mathrm{C}$ \\
$22^{\circ} \mathrm{C}$ \\
$37^{\circ} \mathrm{C}$ \\
Growth in \% NaCl: \\
$0 \%$ \\
$3 \%$ \\
$6 \%$ \\
$8 \%$ \\
Growth on TCBS-1 \\
Swarming \\
O/129 sensitivity \\
G: \\
\hline . \\
\hline
\end{tabular}

G: green colonies; +: positive; -: negative; F: fermentative; NT: not tested.

ATCC: American Type Culture Collection. 
chemical characterization clearly includes the bacterial isolates collected from affected Asian seabass kidneys in the genus Photobacterium on the basis of the following characteristics: Gram-negative rod cocci, motile organism, ability to accumulate polyhydroxybutirate as an intracellular reserve product, ability to grow at a high temperature $\left(35^{\circ} \mathrm{C}\right)$, production of arginine dihydrolase, inability to produce alginase, gelatinase, and indole, utilization of D-mannitol, and requirement of over $100 \mathrm{~mm}$ $\mathrm{Na}^{+}$for optimal growth ${ }^{19)}$. More deeply biochemical characterization has allowed to include the isolated strains as $P$. damselae subsp. damselae according to the criteria established by Holt et al. ${ }^{14)}$, based on the ability to grow at $1-6 \% \mathrm{NaCl}$, gas production from glucose, production of urease and nitrate reductase, and lack of gelatinase and ornithine decarboxylase activities (Table 1). This result was confirmed using the API-20E system where the numerical code obtained was 601400417 at $99.9 \%$ confidence level, and similar to that of the $P$. damselae subsp. damselae reference strain (601500417).

Although the utilization of commercial identification kit has been proved as useful and rapid tool for bacteria of clinical source, including human ${ }^{20)}$, in the case of environmental strains is necessary the confirmation based on serological and/or molecular techniques. Two separate PCR amplifications were employed to identify the bacterial isolates as $P$. damselae subsp. damselae. Samples corresponding to $P$. damselae subsp. damselae yielded two amplification fragments corresponding to 16S rRNA (267 bp) and ureC (448 bp) genes (Fig. 2).

The $P$. damselae subsp. damselae strain isolated in this study from Asian seabass produced $\beta$-hemolysis,

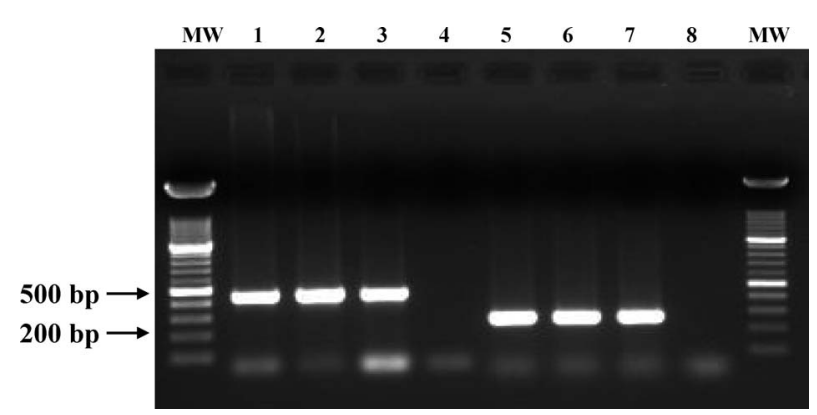

Fig. 2. Agarose electrophoresis of the PCR products for ureC (Lanes 1-4) and 16S rRNA genes (Lanes 5-8) obtained from Asian seabass isolate LCA 24907 and different reference strains used as positive controls. Lanes: MW, molecular weight marker 100 bp Molecular Ruler (Bio-Rad); 1 and 5, Photobacterium damselae subsp. damselae ATCC 33539; 2 and 6, Photobacterium damselae subsp. damselae CECT 5064 strains amplifications for ure $C$ and 16S rRNA genes, respectively; 3 and 7 , Asian seabass isolate LCA 24907 for ure C and 16S rRNA genes, respectively; 4 and 8 , Negative controls (PCR Master Mix without DNA). that according to Fouz et al. ${ }^{21)}$ may be considered as a potencial virulence factor of this microorganism. The $\mathrm{LD}_{50}$, determined by Asian seabass with IP inoculation was $8.1 \times 10^{5} \mathrm{CFU} / \mathrm{g}$ of fish weight. This $L_{50}$ may be considered as moderate virulence, according to the Santos et al. ${ }^{22)}$ criteria that correspond to a strain with moderate virulence. Similar results have been obtained for $P$. damselae subsp. damselae isolated from cultured fish, such as turbot Scophthalmus maximus and rainbow trout Oncorhynchus mykiss ${ }^{2}$, redbanded seabream Pagrus auriga ${ }^{5)}$, and cultured shrimps ${ }^{6,23)}$.

In this study, the isolation, characterization and pathogenicity of $P$. damselae subsp. damselae from cultured Asian seabass in Thailand showing abdominal swelling are described. On the basis of the resistance degree of this strain to the antimicrobials approved by the Food and Drug Administration for use in humans and food aquatic animals (data not shown), the persistence of this bacterium may pose a serious threat to the aquaculture industry in Southeastern Asia.

\section{Acknowlegements}

This study has been supported in part by the Project RTA2005-00028 from INIA (Subprograma Nacional de Recursos y Tecnologías Agrarias en cooperación con las Comunidades Autónomas) from the Spanish government. $\mathrm{MM}$ is the recipient of a research contract from INIA (Spanish Ministry of Science and Technology) cofunded by the European Social Funds.

\section{References}

1) Vera, P., J. I. Navas and B. Fouz (1991): Bull. Eur. Ass. Fish Pathol., 11, 112-113. 2) Fouz, B., J. L. Larsen, B. Nielsen, J. L. Barja and A. E. Toranzo (1992): Dis. Aquat. Org., 12, 155166. 3) Sung, H-H, H-C Li, F-M Tsai, Y-Y Ting and L. Chao (1999): J. Exp. Mar. Biol. Ecol., 236, 261-271. 4) Hosseini, H., A. M. Cheraghali, R. Yalfani and V. Razavilar (2004): Food Contr., 15, 187-190. 5) Labella, A., M. Vida, M. C. Alonso, C. Infante, S. Cardenas, S. Lopez-Romalde, M. Manchado and J. J. Borrego (2006): J. Fish Dis., 29, 175-179. 6) Vaseeharan, B., S. Sundararaj, T. Murugan and J. C. Cheng (2007): Lett. Appl. Microbiol., 45, 82-86. 7) Ghinsberg, R. G., V. Drasinover, Y. Sheinberg and Y. Nitzan (1995): Biomed. Lett., 51, 151-159. 8) Fouz, B., A. E. Toranzo, E. Marco-Noales and C. Amaro (1998): FEMS Microbiol. Lett., 168, 181-186. 9) Fouz, B., A. E. Toranzo, M. Milan and C. Amaro (2000): J. Appl. Microbiol., 88, 531-535. 10) Clarridge, J. E. and S. Zighelboim-Daum (1985): J. Clin. Microbiol., 21, 302-306. 11) Shin, J. D., M. G. Shin, S. P. Suh, D. W. Ryang, J. S. Rew and F. S. Nolte (1996): Clin. Infect. Dis., 22, 856-857. 12) Fraser, S. L., B. K. Purcell, B. Delgado, Jr., A E. Baker and A. C. Whelen (1997): Clin. Infect. Dis., 25, 935-936. 13) Azad, I. S., A. R. Thirunavukkarasu, M. Kailasam and J. J. S. Rajan (2004): Asian Fish. Sci., 17, 1-4. 14) Holt, J. G., N. R. Krieg, P. H. A. Sneath, J. T. Staley and S. T. Williams (1994): Bergey's manual of determinative bacteriology. Williams and Wilkins Co., Baltimore. 15) Osorio, C. R., A. E. Toranzo, J. L. Romalde and J. L. Barja (2000): Dis. Aquat. Org., 40, 177-183. 16) Balebona, 
M. C., I. Zorrilla, M. A. Moriñigo and J. J. Borrego (1998): Aquaculture, 166, 19-35. 17) Zorrilla, I., M. C. Balebona, M. A. Moriñigo, C. Sarasquete and J. J. Borrego (1999): J. Fish Dis., 22, 167-172. 18) Garcia-Rosado, E., I. Cano, B. MartinAntonio, A. Labella, M. Manchado, M. C. Alonso, D. Castro and J. J. Borrego (2007): Int. Microbiol., 10, 193-199. 19) Gauthier, G., B. Lafay, R. Ruimy, V. Breittmayer, J. L. Nicholas, M. Gauthier and R. Christen (1995): Int. J. Syst. Bacteriol., 45,
139-144. 20) O'Hara, C. M., E. G. Sowers, C. A. Bopp, S. B. Duda and N. A. Strockbine (2003): Clin. Microbiol., 41, 56545659. 21) Fouz, B., J L. Barja, C. Amaro, C. Rivas and A. E. Toranzo (1993): Curr. Microbiol., 27, 341-347. 22) Santos, Y., A. E. Toranzo, J. L. Barja, T. P. Nieto and T. G. Villa (1988): Infect. Immun., 56, 3285-3293. 23) Song, Y. L., W. Cheng and C. H. Wang (1993): J. Invertebr. Pathol., 61, 24-31. 